This item was submitted to Loughborough's Research Repository by the author.

Items in Figshare are protected by copyright, with all rights reserved, unless otherwise indicated.

\title{
Pendulum limit, chaos and phase-locking in the dynamics of ac-driven semiconductor superlattices
}

PLEASE CITE THE PUBLISHED VERSION

LICENCE

CC BY-NC-ND 4.0

\section{REPOSITORY RECORD}

Alekseev, Kirill N., and F.V. Kusmartsev. 2019. "Pendulum Limit, Chaos and Phase-locking in the Dynamics of Ac-driven Semiconductor Superlattices". figshare. https://hdl.handle.net/2134/1246. 


\title{
Pendulum limit, chaos and phase-locking in the dynamics of ac-driven semiconductor superlattices
}

\author{
Kirill N. Alekseev ${ }^{1,2 *}$, Feodor V. Kusmartsev ${ }^{3 \dagger}$ \\ ${ }^{1}$ Department of Physical Sciences, P.O. Box 3000, University of Oulu FIN-90014, Finland \\ ${ }^{2}$ Theory of Nonlinear Processes Laboratory, Kirensky Institute of Physics, Krasnoyarsk 660036, Russia \\ ${ }^{3}$ Department of Physics, Loughborough University, Loughborough, LE11 3TU, UK
}

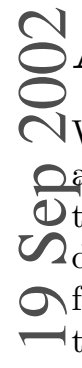

\section{Abstract}

We describe a limiting case when nonlinear dynamics of an ac-driven semiconductor superlattice in the miniband (4) transport regime is governed by a periodically forced and 2 damped pendulum. We find analytically the conditions ᄀor a transition to chaos and consider an influence of temperature on the effect. We also discuss fractional dc voltage states in a superlattice originating from phaseNocked states of the pendulum.

$>$ PACS: $73.21 . \mathrm{Cd} ; \quad 72.20 . \mathrm{Ht} ; \quad 05.45 . \mathrm{a} ; \quad$ 05.45.Ac; m $72.30 .+q$

Keywords: Semiconductor superlattice; pendulum; Cchaos; phase-locking; Josephson junction

\section{Introduction}

Strongly nonlinear dynamical effects in miniband semiI conductor superlattices (SSLs) driven by an intense high$\checkmark$ frequency electric field attract growing interest in the last few years [1, 2, 3, 4, 5, 6, 7, 8, 9]. This theoretical ac$\circlearrowright$ tivity, which includes investigation of dissipative chaos $\because[2,4,6,7$ and a spontaneous generation of dc voltage in 7 a purely ac-driven SSL 1, 3, 4, 5, 7, 8, 9, 10, has been - stimulated by a recent progress in the experimental studies of nonlinear electron transport in the SSLs driven by $\mathrm{THz}$ field [11]. On other hand, recent experiments 12] reveal an important role of coherent plasma oscillations in nonlinear dynamics of carriers in SSLs.

The counterparts of the effects of chaos and spontaneously dc voltage generation in the $\mathrm{THz}$-driven SSL are known in the rf-driven Josephson junctions for a rather long time [13, 14, 15, 16, 17] (for a review, see [18]). Socalled an inverse ac Josephson effect (a quantized dc voltage generation) 16, 17 has already found applications in the design of a zero-biased Josephson voltage standard [18], which offers several advantages in comparison with the conventional voltage standards based on an ac Josephson effect 19. A transition to dissipative chaos often limits the stable operation of such kind of voltage standard [18]. Nonlinear dynamics of driven plasma oscillations in a junction is well-described by the fairly pop-

\footnotetext{
*E-mail: Kirill.Alekseev@oulu.fi

${ }^{\dagger}$ E-mail: F.Kusmartsev@lboro.ac.uk
}

ular Resistively Shunted Junction (RSJ) model [20, 21], which is equivalent to a periodically forced and damped pendulum - one of the most important paradigms in the modern nonlinear science 22].

Within the semiclassical approach [23, ac-driven plasma oscillations in a single miniband SSL are described by the set of balance equations for the average electron velocity (current), the average electron energy and the electric field (voltage) generated by an electron motion in SSL 11, 2, 3, 7, 8, 9]. It has been shown in ref. [2] that this set of three nonautonomous nonlinear first-order differential equations is isomorphic to the periodically forced Lorenz model 24. In comparison with the RSJ model, this system is more complex from a viewpoint of nonlinear dynamics and until recently it has been analysed mainly numerically 1, 2, 3, 4, 7, 9.

However, it should be noticed that a pendulum-like behavior of miniband electrons in SSL has been established earlier for some limiting cases. First, plasma oscillations in SSL have been described within a collisionless approximation by a pendulum without damping in [25, 26, 2]. Second, describing the frequency-to-voltage conversion in ac-driven SSL, Dunlap et al. 27 have found that their model of SSL demonstrates a behavior similar to the phase-locked solutions of damped pendulum [16 for $\omega \gtrsim \omega_{\mathrm{pl}}\left(\omega\right.$ and $\omega_{\mathrm{pl}}$ are the ac electric field frequency and the miniband plasma frequency, correspondently). These earlier findings indicate that dynamics governed by a pendulum can be underlying for nonlinear dynamics of ac-driven SSL.

In this work, we show that the driven damped pendulum could describe a stationary nonlinear dynamics of an ac-driven SSL in the limit when a characteristic scattering constant of electrons with impurities and phonons, $\gamma$, is less than an an ac frequency $\omega$. In contrast to the RSJ model, where the voltage across junction is proportional to the velocity of pendulum, the voltage across SSL depends on both the coordinate and the velocity of pendulum. These findings possess us to apply several exact and approximate results obtained earlier for a pendulum to an analytic investigation of regular and chaotic dynamics in SSL. Namely, we will show the following:

(1) Chaotic dynamics is natural for a relatively low damping, i.e. for $\gamma / \omega_{\mathrm{pl}} \ll 1$.

(2) The SSLs with wide minibands are more stable 
against perturbations leading to a transition to chaos in comparison to the case of narrow miniband SSLs.

(3) The Melnikov criterion of transition to chaos in the pendulum qualitatively explains the position of low frequency boundary of transition to chaos in SSL.

(4) For a relatively low damping, $\gamma / \omega_{\text {pl }} \ll 1$, phaselocking in driven pendulum results in a generation of dc bias across of SSL; the dc voltage per period of SSL is approximately proportional to a half-integer multiplier of $\hbar \omega$.

(5) The difference between generated dc voltage per superlattice's period and $\hbar \omega$ is proportional to $\left(\gamma / \omega_{\mathrm{pl}}\right)^{2}$ at $\gamma / \omega_{\text {pl }} \ll 1$.

(6) For a fairly strong scattering, phase-locking in driven pendulum may not result in a generation of quantized (or an approximately quantized) dc bias across SSL.

Our present analytic research explains and further develops the results obtained numerically in the previous publications [2, 3, 4, 9].

\section{Pendulum limit in superlattice balance equations}

We consider electron transport through a single miniband of a spatially homogeneous SSL under the influence of ac current. We assume the tight-binding dependence $\varepsilon(k)=\Delta / 2[1-\cos (k a)]$ of the electron energy $\varepsilon$ on its quasimomentum $k$ along SSL's axis, where $\Delta$ is the miniband width, and $a$ is the period of SSL. The electron dynamics could be described by the following balance equations [1, 2, 3, 8, 8, 9] (we use notations of ref. [9])

$$
\begin{aligned}
\dot{v} & =u w-\gamma v \\
\dot{w} & =-u v-\gamma\left(w-w_{\mathrm{eq}}\right) \\
\dot{u} & =\omega_{0}^{2} v+I_{0} \sin \omega t
\end{aligned}
$$

Here the scaled variable $u=e a E(t) / \hbar$ describes the electric field $E$ (or the voltage per SSL's period), generated by an electron motion along the SSL axis; $v=m_{0} \bar{V} a / \hbar$ is the scaled electron velocity ( $\bar{V}$ is the electron velocity averaged over the time-dependent distribution function satisfying the Boltzmann equation, $m_{0}=\frac{2 \hbar^{2}}{\Delta a^{2}}$ is the effective electron mass at the bottom of miniband) and $w=(\bar{\varepsilon}-\Delta / 2)(\Delta / 2)^{-1}$, where $\bar{\varepsilon}$ is the average electron energy within the miniband. Following this scaling for the energy, the lower (upper) edge of the miniband corresponds to $w=-1(w=+1)$. Next, $\omega$ is the frequency and $I_{0}$ is the scaled amplitude of driving ac current; $\gamma$ is the phenomenological relaxation constant describing different channels of electron scattering in SSL; $\omega_{0}=\omega_{\mathrm{pl}}\left(C_{\mathrm{sl}} / C\right)^{1 / 2}$ is the generalized plasma frequency, where $\omega_{\mathrm{pl}}=\left(4 \pi e^{2} N / m_{0} \epsilon_{0}\right)^{1 / 2}$ is the miniband plasma frequency, $N$ is the number of electrons per unit volume, $\epsilon_{0}$ is an average dielectric constant for the SSL material, $C_{\mathrm{sl}}=\frac{\epsilon_{0} S}{4 \pi l}$ is the self-capacitance of SSL with the length $l$ and the cross-section area $S$, and $C$ is a parasitic capacitance, which is in parallel to the SSL's capacitance.
The electron energy within miniband relaxes to the thermal equilibrium energy value, $w_{\text {eq }}\left(w_{\text {eq }} \leq 0\right)$; in the case of a nondegenerate electron gas, $w_{\text {eq }}$ has the following temperature dependence 30, 31.

$$
w_{\mathrm{eq}}=-\frac{I_{1}\left(\Delta / 2 k_{B} T\right)}{I_{0}\left(\Delta / 2 k_{B} T\right)},
$$

where $I_{0,1}$ are the modified Bessel functions, $T$ is the lattice temperature, and $k_{B}$ is the Boltzmann's constant. Note that the dependence (2) well describes temperature-induced modifications of the Drude conductivity in the narrow miniband SSLs as have been observed in the experiments [28, 29].

First two equations of the set (1) are the well-known balance equations of Ignatov and Romanov [30, 31, which could be derived from the Boltzmann transport equation with a constant relaxation time $\gamma$. Thus, instead of finding the time-dependence of distribution function $f(p, t)(p=\hbar k)$ from the Boltzmann equation and further calculation of the average electron velocity $\bar{V}=\int d p f(p, t) \partial \varepsilon(p) / \partial p$ and the average electron energy $\bar{\varepsilon}=\int d p f(p, t) \varepsilon(p)$, it is possible just to solve the corresponding balance equations for the average variables. Both these approaches give the same result for the tightbinding dispersion relation.

The third equation in (11) could be obtained from the Maxwell or Kirchoff equations [1, 2, 3, 7, 8]. To the best our knowledge, three coupled balance equations in the form (11) have appeared first in the work of Tetervov [32], who used these equations for a description of decay of undriven $\left(I_{0}=0\right)$ nonlinear plasma oscillations in SSL.

Now we want to show how the pendulum limit arises in the superlattice balance equations (1). We introduce new variables $A$ and $\theta$ as $w=-A \cos \theta, v=-A \sin \theta$. We can re-write Eqs. (1) in the form (see Appendix A)

$$
\begin{aligned}
\ddot{\theta}+ & A \omega_{0}^{2} \sin \theta+\gamma G(\theta, A) \dot{\theta}= \\
& I_{0} \sin \omega t+\gamma^{2} w_{\mathrm{eq}} A^{-1} F(\theta, A), \\
\dot{A}= & -\gamma A-\gamma w_{\mathrm{eq}} \cos \theta,
\end{aligned}
$$

where

$$
\begin{aligned}
G(\theta, A) & =-w_{\mathrm{eq}} A^{-1} \cos \theta \\
F(\theta, A) & =\sin \theta+w_{\mathrm{eq}} A^{-1} \sin \theta \cos \theta \\
\dot{\theta}(t) & =u(t)+\gamma w_{\mathrm{eq}} A^{-1}(t) \sin \theta(t) .
\end{aligned}
$$

Formally, the set of equations (3)-(7) is not simpler than the original balance equations (1). However, this form is more suitable for the consideration of pendulum limits.

We start our analysis with the case of collisionless SSL, $\gamma=0$. As follows from Eq. (4), $A^{2}=v^{2}(t)+w^{2}(t)$ is the integral of motion in this case. For natural initial conditions $v(0)=0, w(0)=w_{\text {eq }}$, we have $A=-w_{\text {eq }}$. The balance equations are reduced to single equation of undamped and periodically forced pendulum

$$
\ddot{\theta}+\left(-w_{\text {eq }}\right) \omega_{0}^{2} \sin \theta=I_{0} \sin \omega t,
$$


where $v(t)=w_{\text {eq }} \sin \theta(t), w(t)=w_{\text {eq }} \cos \theta(t), u(t)=$ $\dot{\theta}(t)$. Earlier the same equation has been obtained within the semiclassical Hamiltonian approach by several authors 25, 26. It was used in the investigations of nonlinear plasma oscillations 25, the instabilities and the Hamiltonian chaos [26] in ideal ac-driven SSLs.

Eq. (8) is valid only for a short time $t \ll \gamma^{-1}$. Now we want to show that a pendulum equation can also describe electron's dynamics in the ac-driven SSL at long time $\left(t \gg \gamma^{-1}\right)$ providing $\omega \gg \gamma$. Eq. (3) represents a pendulum, parameters of which are varied self-consistently due to their dependences on $A$ and $\theta$. To begin with we limit our attention to the time interval $T=2 \pi / \omega$. During this characteristic period of oscillations (or rotations), $T$, the variable $A$ only lightly decays for $\omega \gg \gamma$ (see Eq. (4)). Now we compare characteristic damping terms in Eqs. (3) and (4). Following Eqs. (3) and (5), the averaged over period of ac field dissipative term is

$$
\langle\gamma G(\theta, A)\rangle=-\gamma w_{\text {eq }}\left\langle A^{-1} \cos \theta\right\rangle
$$

Thus, the ratio of characteristic dissipative terms in Eqs. (3) and in Eq. (4), calculated during the time interval $T$, is following

$$
\frac{\langle\gamma G(\theta, A)\rangle}{\gamma}=-w_{\text {eq }}\left\langle A^{-1} \cos \theta\right\rangle \ll 1
$$

This ratio is small because the term $\cos \theta$ changes its signs (oscillates), while $A$ stays almost constant. Next, we consider more long time interval of more number of periods $T$ and come to a conclusion that a dissipation in Eq. (1) is, in effect, much more strong than a dissipation in Eq. (3); the variable $A(t)$ approach its stationary value faster than the variable $\theta(t)$ approaches its attractor. Therefore, if additionally the amplitude of driving current is not large, $I_{0} / \omega_{0}^{2}<1$, the variable $A$ can be adiabatically eliminated [33]. That is, we can substitute the stationary value $A(t) \rightarrow A_{\mathrm{st}}(t)=-w_{\mathrm{eq}} \cos \theta$ in Eq. (3). Observing that $G\left(\theta, A_{\mathrm{st}}\right)=1$ and $F\left(\theta, A_{\mathrm{st}}\right)=0$, we find that Eqs. (3), (7) take the form

$$
\begin{gathered}
\ddot{\psi}+\gamma \dot{\psi}+\left(-w_{\text {eq }}\right) \omega_{0}^{2} \sin \psi=2 I_{0} \sin \omega t, \\
u=\dot{\psi} / 2+\gamma \tan (\psi / 2),
\end{gathered}
$$

where we introduced $\psi(t)=2 \theta(t)$. This pendulum representation of superlattice balance equations constitutes the main result of this paper.

It is useful to compare some well-known solution of Boltzmann equation for the tight-binding lattice and the corresponding solution of pendulum equation (9). This test can be simplified employing an analogy with the RSJ model of Josephson junctions, which is also governed by a periodically driven and damped pendulum. Consider a SSL driven by a given ac electric field $E=E_{0} \cos \omega t$ with the frequency being much greater than the miniband plasma frequency, $\omega \gg \omega_{0}$. In this case we have $I_{0}=\omega_{s} \omega$ with $\omega_{s}=e E_{0} a / \hbar$ [2, 7, 8, 9]. For such $I_{0}$ and $\omega$, we find that the solution of pendulum equation for the variable $v(t)=\sin \theta \cos \theta$ is same as the well-known expression for the time-dependent electron's velocity $v(t)$ derived from the exact solution of the Boltzmann equation [30, 34], if $\omega \gg \gamma$ and $I_{0} / \omega_{0}^{2}<1$ (see Appendix B). Therefore, we see that the pendulum representation, Eqs. (9)-(10), gives same result as the Boltzmann equation within the range of its validity. Moreover, this range of parameters, $I_{0} / \omega_{0}^{2}<1$ and $\omega \gg \omega_{0}$, corresponds to $z \equiv \omega_{s} / \omega \ll 1$ for $\omega \gg \omega_{0}$, what are quite typical values of the field strengths and the frequencies in the modern experiments on harmonics generation and detection of $\mathrm{THz}$ radiation in SSLs [11]. Finally, it is worth to notice that from the viewpoint of analogy with the Josephson junctions, a described generation of harmonics of an ac electric field in SSL is completely equivalent to the standard ac Josephson effect [18, 21], i.e. to a generation of current's harmonics under the action of a sinosuidal voltage.

In this paper we are mainly focused on nonlinear dynamical effects arising within the pendulum limit. With the reference to the pendulum representation of balance equations, we can make the important conclusion: The thresholds of instabilities in an ac-driven semiconductor superlattices, including the transition to chaos, are same as in the RSJ model of an ac-driven Josephson junction providing $\omega \gg \gamma$.

Before we proceed with a consideration of instabilities and chaos, we should note that the SSL balance equations (3)-(7) can be also simplified in the opposite limiting case of frequent collisions, $\omega \ll \gamma$. Really, assuming $|\dot{\theta}| \simeq \omega \ll \gamma$ we see from Eq. (4) that $A(t)$ follows $\theta(t)$ adiabatically. Therefore Eqs. (3), (5), (6), (7) take the form

$$
\gamma \dot{\psi}+\omega_{0}^{2} \sin \psi=2 I_{0} \sin \omega t
$$

with $u=\gamma \tan (\psi / 2)$. Such kind of the first order equation is well-known in the theory of Josephson junctions [35. 21]. Importantly, it also arises in the description of an interaction of electromagnetic radiation with the lateral SSLs [10, 36]. Chaos is impossible in the first order differential equation 22]. However, the overdamped pendulum (11) driven by a two-frequency quasiperiodic force still can demonstrate very complex dynamics known as a strange nonchaotic attractor [37.

Now we turn to the consideration of conditions for a transition to chaos and an influence of temperature effects using the pendulum representation of balance equations (9), (10).

\section{Periodicity and chaos}

The theorem of Levi 38 states that the stationary dynamics of a periodically forced pendulum is never chaotic in the overdamped limit, which in our case reads

$$
C \equiv \frac{\gamma}{\left|w_{\mathrm{eq}}\right|^{1 / 2} \omega_{0}}>2
$$


The numerical simulations of the driven damped pendulum performed for the wide ranges of $I_{0}$ and $\omega$ reveal the absence of chaos already for $C \approx 1$ 39].

For a narrow miniband at room temperature, the thermal equilibrium energy becomes close to the center of miniband, i.e. $\left|w_{\text {eq }}\right|$ is small (see Eq. (2)). Therefore, the criterion for absence of chaos (19) can be easier satisfied in the SSLs with narrow miniband in a comparison to the case of wide miniband SSLs. In particular, for $k_{B} T \gg \Delta$ we have $w_{\text {eq }} \approx-\Delta / 2 k_{B} T$ and therefore $C \propto \gamma / \Delta$ (really, $\omega_{0} \propto m_{0}^{1 / 2} \propto \Delta^{1 / 2}$ providing $\left.\left|w_{\text {eq }}\right|^{1 / 2} \omega_{0} \propto \Delta\right)$. Thus, with a decrease of miniband width $\Delta$, the value of $C \propto \gamma / \Delta$ increases making the condition (12) to be more robust.

As an example we consider the narrow miniband SSL with $\Delta=3 \mathrm{meV}, a=22 \mathrm{~nm}, N=2 \times 10^{15} \mathrm{~cm}^{-3}$ 28 and $\gamma^{-1} \approx 0.5 \mathrm{ps}$ [29], the miniband plasma frequency is $\omega_{\mathrm{pl}}=2.1 \times 10^{12} \mathrm{rad} / \mathrm{sec}$ and $\gamma / \omega_{\mathrm{pl}} \approx 1$. For the temperature $T=50 \mathrm{~K}, w_{\mathrm{eq}} \approx-0.33$ and the factor $\left|w_{\mathrm{eq}}^{-1 / 2}\right|$ is 1.7. For the higher temperature, $T=200 \mathrm{~K}$, $\left|w_{\text {eq }}^{-1 / 2}\right| \approx 3.5$ and chaos is always impossible. However, chaos is still possible for a wider miniband and a longer relaxation time (see corresponding estimates in Refs. [2, 4, 芩, 91).

The criterion of absence of strange attractor, Eq. (12), has been obtained within the pendulum limit and thus is formally valid only for $\omega \gg \gamma$. However, summing up the results of numerical simulations of the superlattice balance equations, we speculate that strange attractor does not exist at any frequency $\omega$, if the inequality (12) is satisfied.

To find an analytic criterion of a transition to chaos in the pendulum limit, we apply the method of Melnikov [40]. This method has been used by several authors for a determination of the conditions of transition to chaos in the periodically driven pendulum (or for the RSJ model) [15, 18. In our case, the Melnikov's criterion for a transition to chaos is

$$
I_{0}>I_{0}^{\mathrm{cr}}=\frac{2 \gamma \omega_{0}\left|w_{\mathrm{eq}}\right|^{1 / 2}}{\pi} \cosh \left(\frac{\pi}{2} \frac{\omega}{\left|w_{\mathrm{eq}}\right|^{1 / 2} \omega_{0}}\right)
$$

(cf. Eq. (60) in Ref. 18). This formula is derived with the assumption that conditions

$$
\frac{\gamma}{\left|w_{\text {eq }}\right|^{1 / 2} \omega_{0}} \ll 1, \quad \frac{2 I_{0}}{\left|w_{\text {eq }}\right| \omega_{0}^{2}} \ll 1
$$

are satisfied. For $\omega<\omega_{0}$ Eq. (13) takes the form

$$
I_{0}^{\mathrm{cr}}=\frac{2}{\pi} \gamma \omega_{0}\left|w_{\mathrm{eq}}\right|^{1 / 2}
$$

In the case of SSL driven by the ac electric field $E=$ $E_{0} \cos \omega t$, one can get $I_{0}=\omega_{s} \omega$ with $\omega_{s}=e E_{0} a / \hbar$ [2, 7, 8, 9]. Then, the formula (14) gives for the boundary of chaotic region in the $\omega_{s}-\omega$ plane in the form

$$
\omega_{s} \omega=\frac{2}{\pi} \gamma \omega_{0}\left|w_{\mathrm{eq}}\right|^{1 / 2}
$$

Earlier, the low-frequency boundary of chaos in the simple form $\omega_{s} \omega=$ const has been found numerically in the works [2, 4, 41], but has been unexplained until now. Eq. (15) can qualitatively explain this dependence.

\section{Phase-locking in pendulum and dc voltage generation in super- lattice}

Phase-locking is another nonlinear dynamic phenomenon which an ac-driven pendulum can demonstrate along with chaos 22]. In the physics of Josephson junctions, phase-locking at zero bias is known as the inverse ac Josephson effect 16, 17, 18, 19. Here we are discussing the influence of phase-locking in the pendulum on the physical properties of SSL. In the driven pendulum phase-locking means that

$$
\langle\dot{\psi}\rangle=\frac{n}{l} \omega
$$

where $n$ and $l$ are integer numbers and $\langle\ldots\rangle$ stands for the time averaging over the period of ac drive $2 \pi / \omega$ [14, 18. Majority of stable phase-locked states are integer $(n \neq 0, l=1)$; however, a fractional phase-locking $(l>1)$ can also exist. In the case of Josephson junction, the voltage $U$ across the junction is proportional to $\dot{\psi}$; therefore phase-locking results in a generation of quantized dc voltage $\langle U\rangle \propto(n / l) \omega[17,18,21]$.

For the superlattice problem, the voltage is a function of both the velocity and the phase of the pendulum (see Eq. (10)). Therefore, phase-locking in the pendulum (16) determines a generation of dc voltage in the SSL as

$$
\begin{aligned}
\langle u\rangle & =\frac{n}{l} \frac{\omega}{2}\left(1+\frac{\gamma}{\omega_{0}} R_{n l}\right), \\
R_{n l} & =\frac{2 \omega_{0}}{\omega} \frac{l}{n}\left\langle\tan \left(\frac{\psi}{2}\right)\right\rangle .
\end{aligned}
$$

For a low damping $\gamma / \omega_{\text {pl }} \ll 1$, we immedeatly get from (17) the dc voltage $\langle u\rangle \approx(n / 2 l) \omega$. Such kind of almost half-integer dc voltage states in a pure ac-driven SSL have been recently observed in the numerical simulations of balance equations [7, 9].

The formula (17) tells us nothing about a stability of dc voltage states in SSL that correspond to the phaselocked states of the pendulum with different $n$ and $l$. However, numerical simulations demonstrate that the dc voltage states, which are close to the integer states (i.e. to the states with $l=1$ and $n$ being even integer), are more typical then fractional states [3, 9].

What is important, the dc voltage per superlattice period is not exactly $(n / 2 l) \omega$ even for a weak damping. Moreover, a weak dependences of $\langle u\rangle$ on the ac amplitude [4, 41] and the frequency [1, 41] have been found numerically at $\gamma / \omega_{\text {pl }} \ll 1$. The appearance of such kind of dependences within the pendulum limit can be understood, if one takes into an account that $\langle u\rangle$ is a function of both the time-average velocity and of the time-average 
coordinate of pendulum (see Eq. (17)). Phase-locked rotational state of the pendulum can be represented in the form 42, 14,

$$
\psi(t)=\psi_{0}+n \omega+\sum_{p=1}^{\infty} \alpha_{p} \sin \left(p \omega t+\mu_{p}\right),
$$

where $\psi_{0}, \alpha_{p}$ and $\mu_{p}$ are constants and we restrict our consideration to the leading phase-locked states, $\langle\dot{\psi}\rangle=$ $n \omega$. The amplitudes $\alpha_{p}$ and the phases $\mu_{p}$ in Eq. (19) are functions of the main physical parameters of our problem, i.e. $I_{0}, \omega$ and $\gamma$. Substituting the expression (19) in Eq. (18) one can see that the dependence of dc voltage $\langle u\rangle$ on the ac amplitude and frequency arise in Eq. 17 via the term $R_{n l}$.

It is important to know how this "dissipative correction" to the quantized dc voltage, $\langle u\rangle-n \omega / 2=$ $\left(\gamma / \omega_{0}\right) R_{n}$, scales with an increase of dissipation. To estimate the dependence of $R_{n}$ on $\gamma / \omega_{0}$ for several lowest phase-locked states of the pendulum, we consider the limit of fast rotations $\alpha \ll 1$. In this case formula (19) takes the form

$$
\psi(t)=\psi_{0}+n \omega+\alpha \sin (\omega t+\mu) .
$$

Substituting Eq. (20) in Eq. (9) and equating zero harmonics, we have the formula (cf. 14, 42)

$$
\omega_{0}^{2} J_{n}(\alpha) \sin (n \mu)=-n \gamma \omega
$$

which gives us an opportunity to understand the characteristic dependences of $\alpha$ and $\psi$ on $\gamma$ and $\omega$. Next, substituting Eq. (20) in Eq. (18) and calculating integrals in the limit $\alpha \ll 1$, we obtain (see Appendix D)

$$
\begin{aligned}
\langle\tan (\psi / 2)\rangle_{n=1} & =\alpha \sin (\mu)+\mathcal{O}\left(\alpha^{2}\right), \\
\langle\tan (\psi / 2)\rangle_{n=2} & =-0.25 \alpha^{2} \sin (2 \mu)+\mathcal{O}\left(\alpha^{4}\right) .
\end{aligned}
$$

Now combining Eqs. (18), 21), (22) and using the asymptotics of the Bessel functions $J_{n}(z) \approx z^{n} /\left(2^{n} n !\right)$ $(z \ll 1)$, we find

$$
R_{n} \simeq(-1)^{n} \frac{4 \gamma}{\omega_{0}}, \quad(\mathrm{n}=1,2)
$$

Thus, for a weak dissipation $\left(\gamma / \omega_{\mathrm{pl}} \ll 1\right)$, the dc voltage spontaneously generated in a pure ac-driven SSL is

$$
\langle u\rangle=n \omega / 2+\mathcal{O}\left(\gamma^{2} / \omega_{\mathrm{pl}}^{2}\right)
$$

and it has a quadratic dependence on $\gamma$.

With a further increase of $\gamma$, the dissipative term in Eq. (17) becomes more important. Moreover, for a large dissipation the phase-locked rotational states (19), (20) do not exist in the pendulum anymore 14]. However, the unquantized dc voltage still can be generated in SSL due to different mechanisms, as has been demonstrated numerically in [9]. The discussion of relationship between a pendulum dynamics and a dc voltage generation in a superlattice for an arbitrary damping will be presented elsewhere.

\section{Discussion and conclusion}

It is instructive to compare the pendulum equation (9), derived from the superlattice balance equations in the limit of rare collisions $(\gamma \ll \omega)$ and corresponding motion equation that can be obtained employing the Newton law. We introduce simplest form of phenomenological dissipative term to the motion equation for electron's momentum as

$$
\dot{p}+\gamma p=-e E(t)
$$

Further, combining this equation and the standard definition of electron's velocity $V=\varepsilon(p) / \partial p$, the tightbinding dispersion relation, and the Maxwell equation (third equation in the set (1)), we get the pendulum equation in the form

$$
\ddot{\phi}+\gamma \dot{\phi}+\omega_{0}^{2} \sin \phi=I_{0} \sin \omega t,
$$

where $\phi=p a / \hbar,\left|w_{\text {eq }}\right| \equiv 1$ for simplicity. In this approach the scaled voltage is $u=\dot{\phi}$. Certainly, this equation is similar to Eq. (9). The difference arises mainly in the dependence of voltage on coordinate and momentum of the pendulum (cf. Eq. (10)). In particular, the voltage is dependent not only on the momentum of pendulum but also on its coordinate in the Boltzmann equation approach.

The crude approach based on the Newton law uses a trajectory of an individual electron. Therefore, this approach can not take into an account the changes in a distribution function of electrons induced by an electric field. In contrast, the approach based on the Boltzmann equation does include such kind of corrections in the term proportional to $\gamma$. In the limit of infrequent collisions, $\gamma \ll \omega$, this term can be often ignored because $\dot{\theta} \simeq \omega \gg \gamma$. However, the dissipative contribution to voltage can become important if one considers a dc voltage generation, $\langle u\rangle \neq 0$. As we have demonstrated in section 4 , this dissipative correction can be important in the consideration of phase locking, $\langle\dot{\theta}\rangle=\omega$. The contribution of dissipative term to the dc voltage, $\langle u\rangle$, can be even more important in the case of lateral superlattices, for which $\langle\dot{\theta}\rangle=0$.10.

In summary, we show that pendulum equation can well describe the dynamics of electrons in a miniband of ac-driven semiconductor superlattice in the limit of weak scattering. We demonstrate that several earlier numerical findings concerning chaos and spontaneous dc voltage generation in the superlattices can be explained within such approach. We establish a link between the theory of diffusive transport in ac-driven superlattices and the theory rf-driven Josephson junctions. We point out that dissipative terms arising in the dependence of voltage across superlattice on the momentum and the coordinate of the pendulum should play an important role in many situations.

We believe that described in this work simple and instructive theory can be useful in an explanation of the results of experiments 11, 12 as well as that it can stimulate other experiments on the $\mathrm{THz}$-field induced nonlinear phenomena in superlattices. 


\section{Acknowledgements}

We are grateful to David Campbell, Ethan Cannon, Alexander Zharov, Pekka Pietiläinen for collaboration. We thank Laurence Eaves, Martin Holthaus, Anatoly Ignatov, Karl Renk, Mikko Saarela, Erkki Thuneberg for discussions. This research was partially supported by the Academy of Finland and the Royal Society.

\section{A Appendix.}

In this appendix we will derive the equations (3), (4), (5), (6), (77). To begin with we introduce a complex variable $Z=w+i v$ and represent the superlattice balance equations (11) in the form

$$
\dot{Z}=-i u Z-\gamma Z+\gamma w_{\text {eq }} .
$$

Substituting the parametrization $Z=-A \exp (i \theta)$ (i.e. $w=-A \cos \theta$ and $v=-A \sin \theta)$ in (25) and equating real and imaginary parts, we get

$$
\begin{aligned}
\dot{A} & =-\gamma A-\gamma w_{\mathrm{eq}} \cos \theta, \\
\dot{\theta} & =u+\gamma w_{\mathrm{eq}} A^{-1} \sin \theta .
\end{aligned}
$$

From the last equation we have

$$
\ddot{\theta}=\dot{u}+\gamma w_{\mathrm{eq}} \frac{d}{d t}\left[A^{-1} \sin \theta\right] .
$$

Taking into account that

$$
\begin{aligned}
& \frac{d}{d t}\left\langle\left[A^{-1} \sin \theta\right\rangle\right]=-A^{-2} \dot{A} \sin \theta+A^{-1} \dot{\theta} \cos \theta \\
& =\gamma A^{-1} \sin \theta+\gamma w_{\mathrm{eq}} A^{-2} \sin \theta \cos \theta+\dot{\theta} A^{-1} \cos \theta
\end{aligned}
$$

and using the third equation of set (1), we immediately get from Eq. (28) the Eqs. (3), (5), (6). Finally, it is easy to see that Eqs. (26) and (27) are same as Eqs. (4) and (7), correspondently.

\section{B Appendix.}

In this appendix we will show that well-known result of Tsu-Esaki-Ignatov-Romanov from the theory of acdriven SSLs 30, 34 can be reproduced within the pendulum representation of the SSL balance equations (9), (10).

Solving the Boltzmann equation for the tight-binding lattice driven by the electric field $E=E_{0} \cos \omega t$ with $\omega \gg \omega_{0}$, Ignatov and Romanov [30] have found for $\gamma \ll \omega$ the following time-dependence of the averaged electron's velocity

$v(t)=\sum_{k=1}^{\infty} a_{2 k-1} \sin (2 k-1) \omega t, \quad a_{2 k-1}=2 J_{0}(z) J_{2 k-1}(z)$,

where $z=\omega_{s} / \omega=\left(e E_{0} a\right) / \hbar \omega$. Same result has been obtained earlier by Tsu and Esaki for the limiting case $z \ll 1$ 34.
Now turn to the pendulum equation. Hence we have the ac field of a very high frequency, we can neglect the last term $(\propto \gamma)$ in Eq. (10) in comparison with the first term $(\propto \dot{\theta} \simeq \omega)$. Further, we can employ an analogy with the RSJ model of Josephson junctions. It is known for the RSJ model that the sinusoidal voltage is the solution of pendulum equation if $\omega \gg \omega_{0}$ and $\gamma \ll \omega$ [18. In our case it means that $\theta(t)=z \sin \omega t$ is the solution of Eq. (9). Further, substituting this time dependence of $\theta(t)$ in the expression for averaged electron's velocity $v=\sin \theta \cos \theta$, we can determine the current generated in the SSL under the action of the ac field. (Of course, it is the analog of the ac Josephson current 18]). Using the Bessel expansions

$$
\begin{aligned}
& \cos (z \sin t)=J_{0}(z)+2 \sum_{k=1}^{\infty} J_{2 k}(z) \cos 2 k t, \\
& \sin \left(z \sin t=2 \sum_{k=1}^{\infty} J_{2 k-1}(z) \cos (2 k-1) t\right.
\end{aligned}
$$

we find the averaged electron's velocity in the form

$$
\begin{aligned}
& v(t)=2 J_{0}(z) \sum_{k=1}^{\infty} J_{2 k-1}(z) \sin (2 k-1) \omega t \\
& +2 \sum_{k_{1}, k_{2} \geq 1} J_{2 k_{1}}(z) J_{2 k_{2}-1}(z) \times \\
& {\left[\sin \left(2\left(k_{2}-k_{1}\right)-1\right) \omega t+\sin \left(2\left(k_{2}+k_{1}\right)-1\right) \omega t\right] .}
\end{aligned}
$$

Supposing that

$$
\omega_{s}<\frac{\omega_{0}^{2}}{\omega}
$$

we have

$$
z \equiv \frac{\omega_{s}}{\omega}<\frac{\omega_{0}^{2}}{\omega^{2}} \ll 1
$$

because $\omega / \omega_{0} \gg 1$. Now it is easy to see that for $z \ll 1$ Eq. (31) is same as Eq. (30).

Thus, we have showed that for $\gamma \ll \omega$ and, additionally, if the condition (32) is satisfied, the pendulum equation can reproduce the result of Tsu-Esaki-IgnatovRomanov. Finally, combining the definition $I_{0}=\omega_{s} \omega$ and Eq. (32), we see that the inequality (32) just gives another condition of the validity of the pendulum approach: $I_{0} / \omega_{0}^{2}<1$.

\section{Appendix.}

In this appendix we will derive the equations (22). We need to calculate the integrals

$$
\langle\tan (\psi / 2)\rangle \equiv \frac{1}{2 \pi} \int_{0}^{2 \pi} d x \tan \left(\frac{n x}{2}+\frac{\alpha}{2} \sin (x+\mu)\right),
$$

where $n=1,2$. In the limit $\alpha \ll 1$ we can use the expansions

$$
\begin{aligned}
& \tan \left(\frac{x}{2}+\frac{\alpha}{2} \sin (x+\mu)\right)=\tan \left(\frac{x}{2}\right)+ \\
& \frac{\sin (x+\mu)}{\cos (x)+1} \alpha-\mathcal{O}\left(\alpha^{2}\right),
\end{aligned}
$$




$$
\begin{aligned}
& \tan \left(x+\frac{\alpha}{2} \sin (x+\mu)\right)=\tan (x)+\frac{\alpha}{2} \frac{\sin (x+\mu)}{\cos ^{2}(x)} \\
& -\frac{\alpha^{2}}{4} \frac{\left(-1+\cos ^{2}(x+\mu)\right) \sin (x)}{\cos ^{3} x}+ \\
& \frac{\alpha^{3}}{24} \frac{\left(-1+\cos ^{2}(x+\mu)\right) \sin (x+\mu)\left(2 \cos ^{2} x-3\right)}{\cos ^{4} x} \\
& +\mathcal{O}\left(\alpha^{4}\right) .
\end{aligned}
$$

After integration of the expression (34) and (35) we obtain Eqs. (22).

\section{References}

[1] A. A. Ignatov et al., Z. Phys. B 98 (1995) 187.

[2] K. N. Alekseev et al., Phys. Rev. B 54 (1996) 10625.

[3] K. N. Alekseev et al., Phys. Rev. Lett. 80 (1998) 2669 .

[4] K. N. Alekseev et al., Physica D 113 (1998) 129.

[5] K. N. Alekseev, M. V. Erementchouk and F. V. Kusmartsev, Europhys. Lett. 47 (1999) 595.

[6] J. C. Cao, H. C. Liu and X. L. Lei, Phys. Rev. B 61 (2000) 5546.

[7] Yu. A. Romanov and Yu. Yu. Romanova, JETP 91 (2000) 1033.

[8] Yu. A. Romanov and Yu. Yu. Romanova, Semiconductors 35 (2001) 204; Yu. A. Romanov et al., J. Appl. Phys. 89 (2001) 3835.

[9] K. N. Alekseev et al., Europhys. Lett. 56 (2001) 842.

[10] K. N. Alekseev et al., Eprint cond-mat/0111454, and to be published.

[11] P. S. S. Guimarães et al., Phys. Rev. Lett. 70 (1993) 3792; A. A. Ignatov et al., Ann. der Physik (Leipzig) 3 (1994) 137; B. J. Keay et al., Phys. Rev. Lett. 75 (1995) 4102; K. Unterrainer et al., Phys. Rev. Lett. 76 (1996) 2973; S. Winnerl et al., Phys. Rev. B 56 (1997) 10303; S. Winnerl et al., Appl. Phys. Lett. 73 (1998) 2983; S. Winnerl, Microelectronics J. 31 (2000) 389; S. Winnerl et al., Appl. Phys. Lett. 77 (2000) 1259; E. Schomburg et al., Physica E 7 (2000) 814; F. Klappenberger et al., Appl. Phys. Lett. 78 (2001) 1673.

[12] R. Bratschitsch et al., Physica B 272 (1999) 375; T. Dekorsy et al., Phys. Rev. Lett. 85 (2000) 1080.

[13] B. A. Huberman, J. P. Crutchfield and N. H. Packard, Appl. Phys. Lett. 37 (1980) 750.

[14] D. D'Humieres et al., Phys. Rev. A 26 (1982) 3483.

[15] V. N. Gubankov et al., Sov. Phys. JETP 59 (1984) 198.
[16] D. N. Langenberg et al., Phys. Lett. 20 (1966) 563.

[17] M. T. Levinsen et al., Appl. Phys. Lett. 31 (1977) 776 .

[18] R. L. Kautz, Rep. Prog. Phys. 59 (1996) 935.

[19] C. A. Hamilton, Rev. Sci. Instr. 71 (2000) 3611.

[20] D. E. McCumber, J. Appl. Phys. 39 (1968) 3113; W. C. Stewart, Appl. Phys. Lett. 12 (1968) 277.

[21] K. Likharev, Dynamics of Josephson junctions and circuits, Gordon and Breach, New York, 1986.

[22] R. Z. Sagdeev, D. A. Usikov, G. M. Zaslavsky, Nonlinear Physics: From the Pendulum to Turbulence and Chaos, Harwood Academic Publishers, Chur, Switzerland, 1992.

[23] F. G. Bass and A. P. Tetervov, Phys. Rep. 140 (1986) 237.

[24] C. A. Sparrow, The Lorenz Equations: Bifurcations, Chaos, and Strange Attractors, Springer Verlag, New York, 1982.

[25] E. M. Epshtein, Sov. Phys. Semicond. 12 (1978) 583.

[26] K. N. Alekseev, G. P. Berman and D. K. Campbell, Phys. Lett. A 193 (1994) 54.

[27] D. H. Dunlap et al., Phys. Rev. B 48 (1993) 7975.

[28] G. Brozak et al., Phys. Rev. Lett. 64 (1990) 3163.

[29] A. Sibille et al., Superlatt. Microstr. 13 (1993) 247.

[30] A. A. Ignatov and Yu. A. Romanov, Phys. Stat. Sol. (b) 73 (1976) 327.

[31] A. A. Ignatov, E. P. Dodin and V. I. Shashkin, Mod. Phys. Lett. 5 (1991) 1087; A. A. Ignatov, K. F. Renk and E. P. Dodin, Phys. Rev. Lett. 70 (1993) 1996.

[32] A. P. Tetervov, Izv. VUZ Radiofiz. 27 (1984) 801. (Engl. tranl.: Radiophysics and Quantum Electronics (Consultant Bureau, New York) 27 (1984) 564).

[33] H. Haken, Synergetics-An Introduction, Springer, Berlin, 1977.

[34] R. Tsu and L. Esaki, Appl. Phys. Lett. 19 (1971) 246.

[35] L. G. Aslamazov and A. I. Larkin, JETP Lett. 9 (1969) 150.

[36] E. Vanagas, Lithuanian Phys. J. 32 (1992) 330; E. Vanagas, Electron. Lett. 29 (1993) 777; E. P. Dodin, A. A. Zharov and A. A. Ignatov, JETP 87 (1998) 1226.

[37] A. Bondeson, E. Ott and T. M. Antonsen, Jr., Phys. Rev. Lett. 55 (1985) 2103; F. J. Romeiras et al., Physica D 26 (1987) 277; E. Neumann and A. Pikovsky, Eur. Phys. J. B 26 (2002) 219. 
[38] M. Levi, Phys. Rev. A 37 (1988) 927.

[39] T. H. Yang et al., Phys. Rev. E 51 (1995) 5279.

[40] V. K. Mel'nikov, Trans. Moscow Math. Soc. 12 (1963) 1.

[41] E. H. Cannon, Ph. D. thesis, Department of Physics, University of Illinois at Urbana-Champaign, 1999.

[42] N. F. Pedersen et al., J. Low Temp. Phys. 38 (1980) 1. 